\title{
High-Fidelity Modeling and Control Design for a Cooperative High Altitude Long Endurance Aircraft Landing System
}

\author{
Eduardo Rodrigues Della Noce ${ }^{1}$, Arti Kalra ${ }^{1}$, Andre Coelho ${ }^{1}$, Tin Muskardin ${ }^{1}$ and Konstantin Kondak ${ }^{1}$
}

\begin{abstract}
High Altitude Long Endurance (HALE) aircraft can take flight to altitudes as high as $20 \mathrm{~km}$ and can stay there for long periods of time. In this article, the viability of landing such an aircraft on a mobile platform using a cooperative control strategy for motion synchronization is examined. Time domain system identification is applied to create a model of the Elektra 2 Solar HALE aircraft, which was found to be high fidelity by the Federal Aviation Administration (FAA) standards. An analysis is made to evaluate the feasibility of autonomously landing the HALE Unmanned Aerial Vehicle (UAV) on top of a ground vehicle with a roof-mounted landing platform. Controller synthesis is done for the individual vehicles as well as the cooperative landing control, leading to an examination of the overall system stability and performance, using both deterministic and stochastic methods.
\end{abstract}

\section{INTRODUCTION}

\section{A. Motivation}

There have been many recent projects on High Altitude Platforms (HAPs) and HALE aircraft from both industry and government entities in an attempt to find a viable way of extending or even substituting satellites. This particular type of UAV is designed for stratospheric flight, achieving altitudes in the order of $20 \mathrm{~km}$, and with the use of solar energy generators and appropriate batteries, they can remain airborne for virtually unlimited periods of time. This allows for their use in many different applications, such as earth observation, meteorology and establishing and maintaining communication networks over long periods of time. According to [1], HALE UAV are a promising alternative to satellites due to being cheaper, easier to maintain, and overall more flexible.

Some projects with the objective of creating functional HALE aircraft include Aquila by Facebook [2], Solara-50 by Google [3], Helios by NASA [4], Zephyr by Airbus [5] and ELHASPA by DLR [6], among others. However, today, Airbus is the only company with an operational HAP aircraft (Zephyr), as most of the competition has canceled their research on the topic; the Aquila project was canceled after a structural failure occurred during the landing procedure in its tests [7], and the Solara-50 project met the same fate after also having a prototype crash [8].

As seen in [9], one of the main design goals of HALE aircraft is to maximize aerodynamic efficiency, so that they are able to operate for long periods of time at high altitudes.

\footnotetext{
${ }^{1}$ Eduardo Rodrigues Della Noce, Arti Kalra, Andre Coelho, Tin Muskardin and Konstantin Kondak are with the German Aerospace Center (DLR), Institute of Robotics and Mechatronics, 82234 Wessling, Germany (Email: eduardo.nocedtum.de, arti.kalraedlr.de, andre.coelho@dlr.de, tin.muskardin@gmail.com, konstantin.kondakedlr.de)
}

This translates to high aspect ratios and low airspeed, both measures taken to reduce drag. Because of the low airspeed during flight, the weight of the UAV must be kept low so it can be supported by the lift force, which, together with the aforementioned high aspect ratios, results in a low wing loading and very lightweight structures. All these factors combined generate a couple of challenges, which are still not solved:

- The lightweight design leads to low payload capacities.

- The low airspeed means, especially during landing, that even relatively small wind disturbances can lead to problems such as stall and high crosswind sensitivity (large crab angles at low crosswinds).

- The large dimensions lead to slow rotational dynamics, which not only make it harder to reject turbulence, but also increases the difficulty of performing a de-crab maneuver during landing, raising safety concerns.

To address these issues, a novel landing strategy was proposed in [10], which utilizes a mobile ground vehicle in cooperation with the UAV for the touchdown of the aircraft instead of the use of a landing gear. This is a promising field of research that serves as an alternative way of increasing payload capacity and expanding the conditions in which the aircraft can safely land. According to [11], these two main objectives are achieved in the following way: first, the removal of the landing gear from the aircraft can reduce its weight by about 6 to 15\% [12], thereby increasing the payload capacity; and second, it can increase operational availability, since with a moving platform the UAV is not required anymore to align with the runway direction, which removes the concerns regarding the de-crab maneuver during the final landing phase. In [10] the landing was performed using a Hardware-In-the-Loop (HIL) setup with a virtual aircraft, while in [11] the landing was actually demonstrated with both real vehicles.

There are a number of technical challenges associated with the cooperative control approach [13]:

- It is necessary to identify the optimal landing control strategy for the given vehicle dynamics, where unilateral or bilateral control for each vehicle must be considered, with a possible use of a Model Predictive Control (MPC) strategy as well.

- A high-fidelity model of both aircraft and ground vehicle are necessary for simulation and estimation of control parameters.

- One must identify and implement suitable vehicle controllers, considering, for example, independent control 
of velocity and altitude of the aircraft.

- Precise relative state estimation is necessary for the control of both vehicles to work as intended.

- The cooperative control must work under time delay, especially when considering bilateral control configurations.

- The developed system should be compliant with existing landing procedures.

- Control law clearance methods should be studied with statistical analysis to determine their performance in different landing conditions.

\section{B. Main Contribution}

The work presented in [13], which extends on [10] and [11], presents a thorough analysis of the cooperative landing problem for a demonstrator setup, but as of now, no analysis has been done for an actual HALE aircraft. The demonstrator setup differs from HALE aircraft mainly in size, since the former is a relatively small aircraft, which changes its ground clearance and flying performance in comparison to the latter. In addition, due to its large airframe, the HALE aircraft possesses significantly slower dynamics, imposing a reduced maneuverability and reduced disturbance rejection capabilities. Therefore, a redesign and thorough re-evaluation of the system is required. In this context, this study is a first step towards a proof of concept with the real aircraft.

As such, the main subject of interest in this work is the Elektra 2 Solar $^{2}$ aircraft, and the goal is to analyze the feasibility and performance of its landing on a mobile platform. This requires the development of a high-fidelity model for the aircraft first, which can be achieved with a system identification flight campaign, allowing for the determination of highly accurate model parameters. Currently landing is performed in the side-slip technique and admissible landing conditions are limited to low crosswinds, which is mainly due to the rudder dimensions and wingtip ground clearance. This is because both rudder and aileron are needed to produce a steady side-slip condition (where a bigger rudder would allow for larger side-slip angles), and when landing with the side-slip technique, a certain amount of roll angle is necessary to compensate for the lateral wind force, which reduces the wingtip clearance [13].

The paper is structured as follows. First, a high-fidelity model of Elektra 2 Solar was identified. Data from Inertial Measurement Units (IMUs) were used for the time domain system identification, together with an initial analysis to find a minimal set of parameters that can generate a high-fidelity model of the aircraft.

Second, the high-fidelity Elektra 2 Solar model was used to perform a thorough analysis of its capabilities regarding the landing on a ground vehicle. For this, the simulation framework developed in [13] was adapted and the necessary optimization for the controller gain tuning was performed for the new aircraft. With all gains correctly set for both

\footnotetext{
${ }^{2}$ Elektra Solar, "Elektra Two Solar OPS / UAS." Available at: https://www.elektra-solar.com/products/elektra -two-solar-ops-and-uas.
}

vehicles and the cooperative control strategy, disturbance rejection and landing performance analyses were conducted, using both deterministic and stochastic approaches.

\section{AIRCRAFT MODEL}

In the following sections, details are given in regards to the equations of motion chosen, the propulsion model used, and the model for the aerodynamic forces and moments.

\section{A. Equations of Motion}

The considered equations of motion are based on a rigid body model with 6 degrees of freedom and take aerodynamic, propulsion and gravitational forces and moments into consideration. The non-linear, flat earth equations written in body frame were used [14][15], since they were considered sufficiently precise for the purposes of this work. All aircraft states are inertially referenced (kinematic) quantities, describing the motion of the aircraft with respect to the ground. Furthermore, the aircraft was considered symmetric with respect to the $x z$ plane in body frame.

\section{B. Propulsion}

The propulsion forces and moments were modeled based on data obtained from the propeller manufacturer and static thrust measurements, as proposed in [16] and [17].

\section{Aerodynamics}

When it comes to modeling the aerodynamic forces and moments, system identification was used, which is a strategy that allows the creation of high-fidelity models based on experimental data. The process as a whole can be separated into three main stages: data gathering, identification and validation [18]. For the identification stage in this work, the Two Step Method was used.

For data gathering, elevator pulses were used to excite the phugoid mode of the aircraft. Once the gathered data was extracted and the necessary pre-processing was done (resampling and smoothing of data), the measurements were corrected for sensor biases and drifts, while also being smoothed to eliminate noise, using the Output Error Method, as described in [19].

With the reconstructed forces and moments obtained, it becomes necessary to define a model structure with parameters to be identified using said reconstructions. Ockham's Razor, or the principle of simplicity, is used to define what constitutes a "best model" for an application, and, in translation, that principles reads as: "The number of entities should not be increased beyond what is necessary to explain anything" [20]. As explained in [18], in the case of system identification, "entities" are the hypotheses assumed and the parameters introduced for the creation of the model. Therefore, an extensive investigation was carried out to find a minimal and effective set of aerodynamic derivatives that could make an accurate model for the aerodynamic forces and moments. For the criteria, Theil's Inequality Coefficient (TIC) is used, and a model will be considered adequate when it fulfills the TIC $<0.3$ threshold suggested by [18] 
for all states (in the present case longitudinal velocity $u$, vertical velocity $w$, pitch rate $q$ and pitch angle $\theta$ ). In terms of order of testing and priority of the considered terms, again Ockham's Razor is used, and for simplicity the order will follow Pascal's expansion for polynomials; in other words, first the constant terms, then linear terms, then crossproducts of linear terms, then quadratic terms, and so on. The manual procedure was chosen over an automated one (such as stepwise regression) to gain important insight into the physical properties of the system. It was concluded that the minimal set of parameters for system identification are those shown in equations 1 to 3 .

$$
\begin{aligned}
X & =X_{0}+X_{u} \cdot u+X_{w} \cdot w \\
Z & =Z_{0}+Z_{u} \cdot u+Z_{w} \cdot w+Z_{q} \cdot q \\
M & =M_{0}+M_{u} \cdot u+M_{w} \cdot w+M_{q} \cdot q+M_{\delta e} \cdot \delta e
\end{aligned}
$$

This a classical linear derivative aerodynamics model, where certain terms are missing due to insignificance. Note that this only covers the aerodynamics of the aircraft, and propulsion is modeled as explained before, and not identified. The aerodynamic derivatives are shown in Table I, the statistical measures of states can be found in Table II and Figure 1 illustrate the model validation results for all states.

TABLE I: Elektra 2 Solar aerodynamic derivatives using processed data from flight experiments.

\begin{tabular}{cccccc} 
Term & Value & Term & Value & Term & Value \\
\hline$X_{0}$ & 300.2103 & $Z_{0}$ & -3233.6376 & $M_{0}$ & -540.0559 \\
$X_{u}$ & -47.3480 & $Z_{u}$ & -76.6415 & $M_{u}$ & 15.6499 \\
$X_{w}$ & 26.9232 & $Z_{w}$ & -124.1343 & $M_{w}$ & -12.0922 \\
& & $Z_{q}$ & -8582.7234 & $M_{q}$ & -1747.1684 \\
& & & & $M_{\delta e}$ & -5309.2060
\end{tabular}

For the validation of the model, there are a number of statistical parameters which can be used as performance metrics. Aside from TIC, [18] suggests the use of Goodness of Fit (GOF) as a possible metric for validation of model prediction capabilities; this metric captures how well the model approximates the test data point by point. Other parameters include the coefficient of determination $R^{2}$, the Root Mean Squared Error (RMSE) and the Normalized Root Mean Squared Error (NRMSE).

TABLE II: Averaged statistical measures for the states in model validation of Elektra 2 Solar

\begin{tabular}{c|cccc} 
& \multicolumn{4}{|c}{ Validation Set } \\
\hline Coefficient & $u$ & $w$ & $q$ & $\theta$ \\
GOF & 0.8764 & 0.4040 & 0.8790 & 0.8475 \\
TIC & 0.0197 & 0.1066 & 0.1631 & 0.0943
\end{tabular}

Overall, all TIC are below 0.2 , complying to the established threshold of 0.3 , and all the measures suggest a good agreement between the simulated data and the real data. Furthermore, it can be seen that the period error is less than $10 \%$ and the damping error is less than 0.02 , as shown in Table III; this means the model can be considered high fidelity by FAA's standards [21].
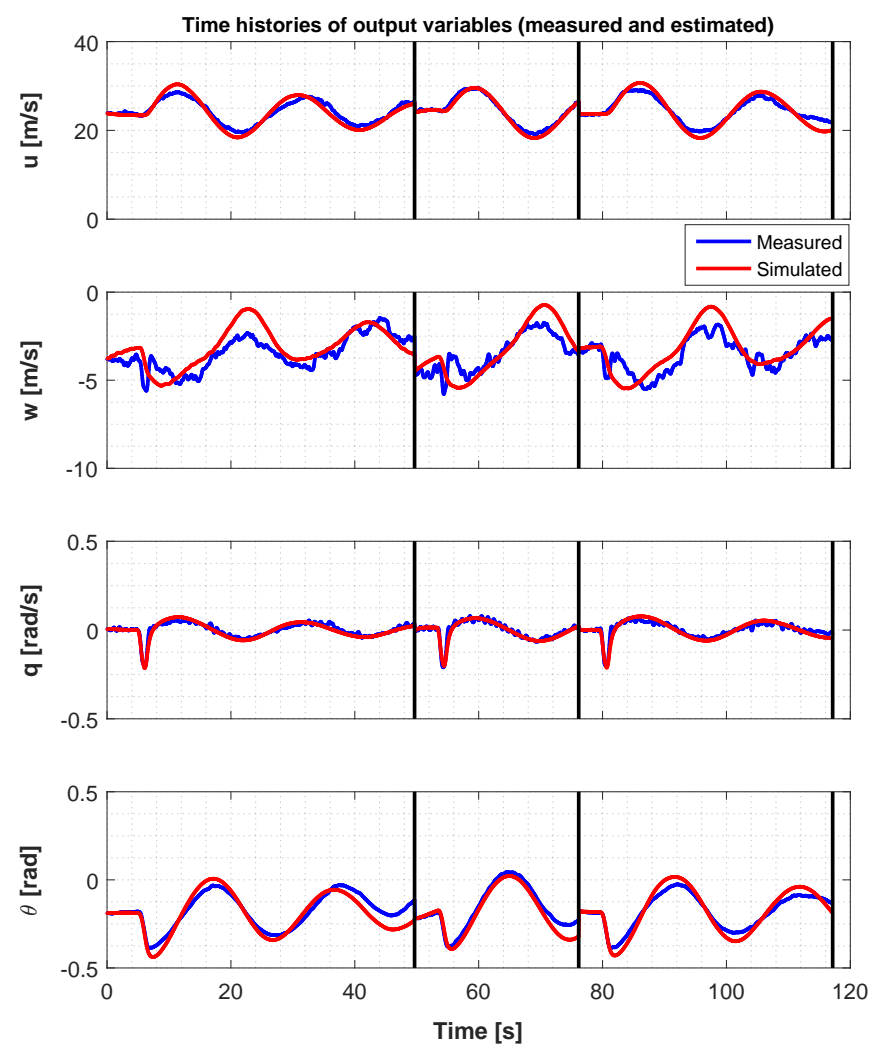

Fig. 1: Model validation of the validation set of Elektra 2 Solar using a forward simulation and data from the first IMU.

TABLE III: Comparison of phugoid characteristics between the identified model and average of flight experiments.

\begin{tabular}{c|c|c|c} 
Criteria & Identified Model & Experiment & Error \\
\hline Period & $19.75 \mathrm{~s}$ & $20.61 \mathrm{~s}$ & $4.1727 \%$ \\
Damping & 0.0599 & 0.0663 & 0.0064
\end{tabular}

Finally, the same procedure was repeated using data from a different, redundant IMU as well. Tables IV and V show the aerodynamic derivatives of Elektra 2 Solar using data from a second IMU and data using an average of both IMUs respectively. Between IMUs 1 and 2, the mean average difference between parameters is $14.82 \%$, with the maximum error being in $X_{w}$, where the difference is $43.51 \%$, while all other errors are lower than $25 \%$. Comparing the parameters from the average of the data to the average of the parameters from both data, the difference has a mean value of $1.41 \%$, with the maximum also being in $X_{w}$, with $5.06 \%$. The only parameters from the identification of the averaged data to not stay within the value range set by the individual identifications are $Z_{w}$ and $M_{w}$, going over their closest boundaries by $0.72 \%$ and $0.51 \%$ respectively. These low differences in values between the generated models suggest the proposed model structure can yield consistent results and has no underlying redundancies. These two new models are also high fidelity by FAA's standards and have TIC $<0.3$ for all states in validation. 
TABLE IV: Elektra 2 Solar aerodynamic derivatives using data from a different IMU.

\begin{tabular}{cccccc} 
Term & Value & Term & Value & Term & Value \\
\hline$X_{0}$ & 258.5348 & $Z_{0}$ & -3084.4334 & $M_{0}$ & -453.4846 \\
$X_{u}$ & -40.4951 & $Z_{u}$ & -82.4015 & $M_{u}$ & 12.7566 \\
$X_{w}$ & 47.6642 & $Z_{w}$ & -122.2783 & $M_{w}$ & -11.8109 \\
& & $Z_{q}$ & -8280.1570 & $M_{q}$ & -1507.1400 \\
& & & & $M_{\delta e}$ & -4727.7932
\end{tabular}

TABLE V: Elektra 2 Solar aerodynamic derivatives using averaged data from the two previous IMUs.

\begin{tabular}{cccccc} 
Term & Value & Term & Value & Term & Value \\
\hline$X_{0}$ & 285.0892 & $Z_{0}$ & -3156.1453 & $M_{0}$ & -496.5808 \\
$X_{u}$ & -44.4189 & $Z_{u}$ & -79.8533 & $M_{u}$ & 14.1648 \\
$X_{w}$ & 35.4966 & $Z_{w}$ & -125.0361 & $M_{w}$ & -12.1543 \\
& & $Z_{q}$ & -8435.2024 & $M_{q}$ & -1629.5471 \\
& & & & $M_{\delta e}$ & -5025.0314
\end{tabular}

\section{GROUND VEHICLE MODEL}

The ground vehicle model consists of a kinematic bicycle model for the lateral direction and a dynamic model for the longitudinal direction (with respect to body frame) [22][23], both of which assume slip-free motion and model the front and rear wheels as single wheels; this is considered a valid approximation for this application with a rather smooth ground vehicle motion. A slow actuation ground vehicle model is used, which represents a semi-autonomous vehicle with a human driver [13].

As for the aerodynamic disturbance caused by the Unmanned Ground Vehicle (UGV), a Computational Fluid Dynamics (CFD) simulation was performed using a realistic geometrical model of the ground vehicle and platform, which generated a vector field representing the change of airflow velocity (magnitude) and angle of attack (direction) induced in the area around it [11].

The use of such landing platform (a car with a horizontal net under tension on top) would be unreasonable for a real application with the Elektra 2 Solar aircraft; in the future, a different design will be necessary to support the weight of the aircraft (possibly being an actuated platform as well), and the final docking mechanism will have to be adapted, but those have not been designed yet. As it stands, this model is enough for the initial investigation of the strategies' feasibility (simulation stops at touchdown).

\section{CONTROL STRUCTURE AND TUNING FOR THE COOPERATIVE LANDING MANEUVER}

The landing procedure has been thoroughly explained in [11], [13]; here, a quick summary is given on the individual vehicles and cooperative control elements of it, which are the parts that were tuned for this specific study.

\section{A. Vehicle Control}

As described in [11], for the aircraft, a classical SingleInput-Single-Output (SISO) controller cascaded in three loops is used for lateral control, while a Multiple-InputMultiple-Output (MIMO) controller is used for both longitudinal and vertical control, based on the Total Energy
Control System (TECS) strategy which was first introduced in [24]. This corresponds to 5 controller gains to be tuned in the lateral direction and 9 in the longitudinal and vertical directions, totaling 14 gains to be tuned for the UAV.

Meanwhile, the ground vehicle is controlled using two PI controllers, one for speed (which commands throttle) and one for course angle (which commands steering wheel deflection). This means there are 4 gains to be tuned for the UGV.

\section{B. Cooperative Control}

In terms of the cooperative control for horizontal positional synchronization, the UAV synchronizes the lateral direction and the UGV synchronizes the longitudinal direction. This method of unilateral action from both vehicles was chosen because it results in an overall system stability which is independent of communication time delays [13].

Figures $2 \mathrm{a}$ and $2 \mathrm{~b}$ show the cooperative control structure that is used in this approach. $C_{X G}, C_{Y A}$ are both Proportional-Integral-Derivative (PID) controllers, the first being for longitudinal control of the UGV and the second for lateral control of the UAV. The dashed feedback loop with a lateral controller for the ground vehicle $C_{Y G}$ is an optional term to realign the UGV with the centerline of the runway after a disturbance. $V_{k, \text { land }}$ is the desired groundspeed during landing.

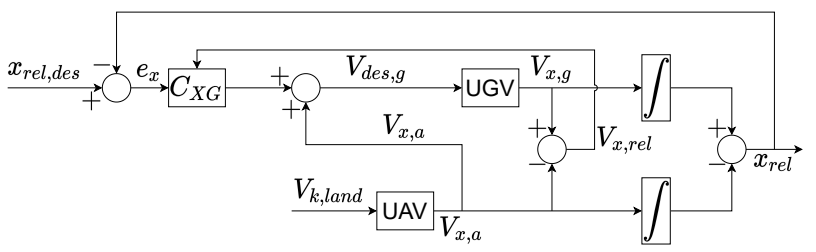

(a) Longitudinal control. Adapted from [13], [25].

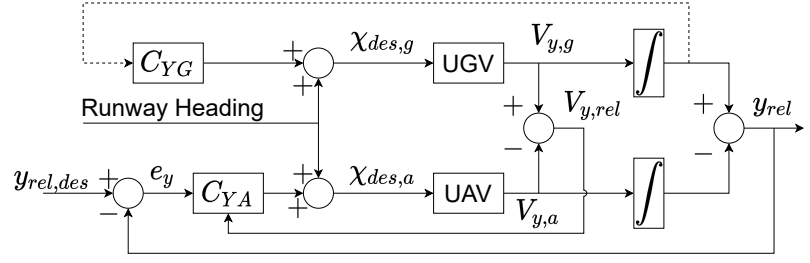

(b) Lateral control. Adapted from [13], [25].

Fig. 2: Structure of the cooperative horizontal synchronization control.

The equations for each vehicle can be written as follows:

$$
V_{d e s, g}=K_{p, g} e_{x}+K_{i, g} \frac{1}{s} e_{x}+K_{d, g} V_{x, r e l}+V_{x, a}
$$

$\chi_{d e s, a}=\operatorname{atan}\left(\frac{K_{p, a} e_{y}+K_{i, a} \frac{1}{s} e_{y}+K_{d, a} V_{y, r e l}}{V_{k, \text { land }}}\right)+\psi_{r n w}$

Here, $K_{p}, K_{i}, K_{d}$ are proportional, integral and derivative gains respectively, subscripts $a$ and $g$ represent values for 
aircraft and ground vehicle respectively, $\psi_{r n w}$ is the runway heading, $V$ and $\chi$ are inertial velocity and course angle respectively, subscript des represents desired value, subscripts $x$ and $y$ represent longitudinal and lateral directions respectively (in the local frame), $e$ is the position error in the considered direction, subscript rel expresses relative quantities. On the derivative part of the PID controllers, the signal is not mathematically derived; instead, the measured velocity error is directly used. This means in total 6 controller gains must be tuned for the cooperative landing controller.

\section{Controller Tuning}

For the controller tuning of the individual vehicles, a time domain design was used with optimization based tuning, using a system step response as reference for the commanded states (groundspeed, altitude, course angle).

For the controller tuning of the cooperative controller, the aircraft begins the simulation $5 \mathrm{~m}$ behind and $5 \mathrm{~m}$ to the left of the UGV, with both having the same groundspeed of $21 \mathrm{~m} / \mathrm{s}$, constituting an initial phase of the optimization of the vehicles aligning themselves without outside interference. At $50 \mathrm{~s}$, a wind gust which consists of a $5 \mathrm{~m} / \mathrm{s}$ headwind and a $5 \mathrm{~m} / \mathrm{s}$ crosswind hits the aircraft, marking the start of the disturbance rejection phase, which also lasts for $50 \mathrm{~s}$. For the entire duration, a communication time delay between aircraft and ground vehicle of $100 \mathrm{~ms}$ is used; this value comes from an investigation conducted in [13], where the time delayed was found to vary between values of $50 \mathrm{~ms}$ and $300 \mathrm{~ms}$, with an average value of around $100 \mathrm{~ms}$. For initial guesses, an investigation is first made with secondorder system models (which are identified using experimental data) for both vehicles across thousands of possible gain values (generated randomly) and the best result is kept as an initial guess for the optimization with the high-fidelity model.

As costs for both cases, well-known metrics such as integral of absolute error (IAE), settling time, overshoot, and control-signal derivative [23][26][27][28] were used. This same tuning procedure has already been used previously to find the gains for the successful landing tests of the Penguin BE UAV as shown in [11], which serves as validation of the method. Results for the cooperative control with the Elektra 2 Solar aircraft with optimal gains are shown in Figure 3.

This leads to a settling time of $9.7 \mathrm{~s}$ and $33.85 \mathrm{~s}$ in longitudinal and lateral direction respectively during the initial displacement phase, and $18.2 \mathrm{~s}$ and $29.4 \mathrm{~s}$ during disturbance rejection. In the same order, it presents overshoots of $1.49 \mathrm{~m}$, $0 \mathrm{~m}, 3.10 \mathrm{~m}$ and $3.12 \mathrm{~m}$.

\section{LANDING PERFORMANCE ANALYSIS}

\section{A. Disturbance Rejection}

With the optimal gains for the horizontal synchronization controllers estimated as described in the previous section, multiple analyses were conducted. For the tests executed here, the geometrical limit of $80 \mathrm{~cm}$ from the center of the landing platform for landing the UAV is carried over from [10], [11] and [13], as it allows for direct comparison with
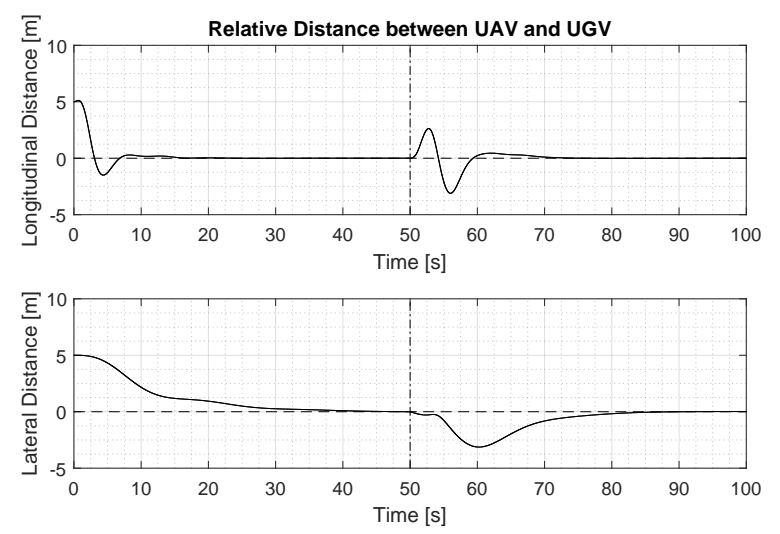

Fig. 3: System response to an initial displacement and a subsequent disturbance at 50 seconds, nominal case; the commanded relative position is set to 0 .

the demonstrator setup from those works, which can grant insights into how the results of a test with a smaller UAV can be translated to HALE aircraft. This limitation can also be seen as a maximum feasible mechanical interface dimension for catching the aircraft.

Like in [13], the disturbance rejection capabilities were evaluated in a deterministic way, where the strongest wind gusts hitting the aircraft from all four main directions without causing a retry of the landing procedure were identified, as shown in Figure 4. These are short wind gusts that last 3 seconds, which can be considered a worst-case scenario for this analysis, given the fact that a discrete wind gust event contains two velocity gradients, while an increase in background wind only contains one. It can be observed that the system is able to reject wind gusts better in the lateral direction than in the longitudinal one, being able to stay within the required landing precision for wind gust magnitudes of up to $2.2 \mathrm{~m} / \mathrm{s}$ and $1.4 \mathrm{~m} / \mathrm{s}$ in the longitudinal and lateral directions, respectively. These values are both lower than the previously observed ones with the smaller UAV in [13], where the maximum tolerable gust disturbances were about $12 \mathrm{~m} / \mathrm{s}$ in the lateral direction and $3.7 \mathrm{~m} / \mathrm{s}$ in the longitudinal direction; this shows, however, the lateral direction rejection is much more influenced by the slower aircraft dynamics than the longitudinal one. To achieve a better accuracy with the slow and large Elektra 2 Solar aircraft, it might be necessary to use a different cooperative control strategy, where the ground vehicle would contribute to both longitudinal and lateral directions, as well as an actuated landing platform. However, these are future research topics.

\section{B. Landing Procedure}

After the disturbance rejection analysis, the nominal landing was studied considering the most important metrics, which are the total runway used and the total time to complete the landing. As can be seen in Figure 5, a total runway length of $683.2 \mathrm{~m}$ was used to complete the maneuver in 41.8 seconds. In Figure 6, which shows the trajectory of the UAV 

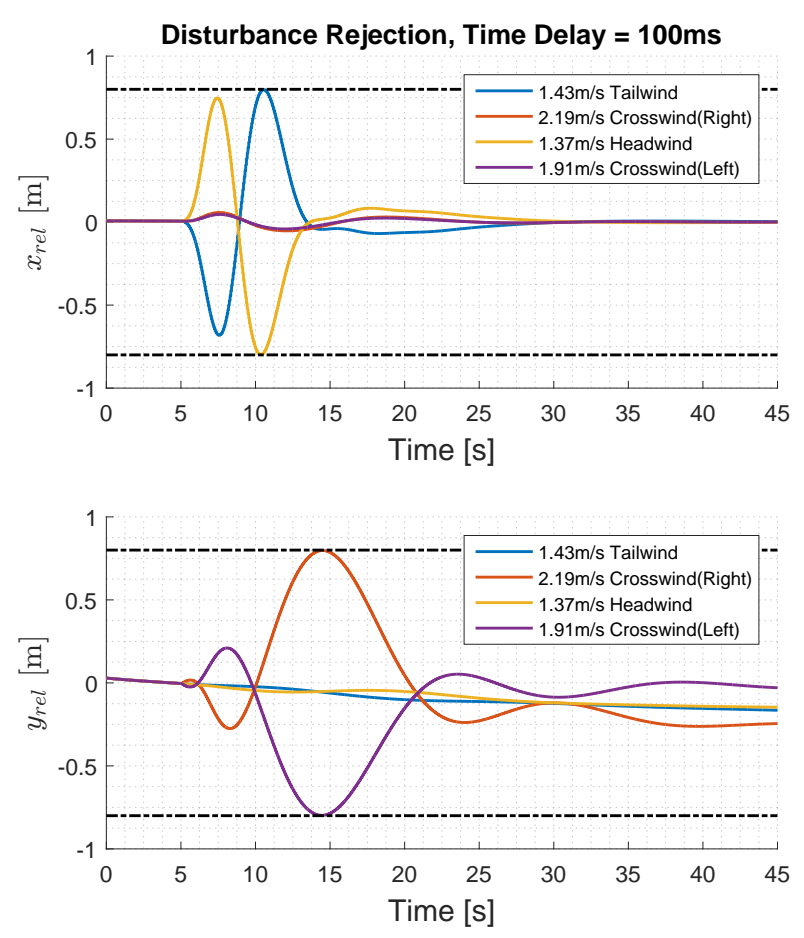

Fig. 4: Maximum wind pulse of 3 seconds in each principal direction to cause a retry for a nominal value $(100 \mathrm{~ms})$ of communication time delay .

relative to the UGV when close to the flare condition, one can see the relative distance in longitudinal direction slightly increases when the position error is almost 0 for the first time; this is due to the switch between linear acceleration mode [11] of the UGV and cooperative control. A few different strategies were tested to diminish this effect, and the best one found was making the change when the UAV and UGV are 5 meters apart. A more thorough analysis should be conducted in the future to further reduce this effect, but this has no impact on further analysis, since the aircraft can enter the flare state without issues. Furthermore, upon entering the "ground lock" state (where ailerons are deflected upwards symmetrically, flaps up, nose down, and elevator is allowed enough control authority for pitch damping upon contact with the net the locking mechanism hooks in), a sudden displacement of the aircraft can be seen due to engine retard, which causes it to lose speed; see more in [11] and [13]. For this maneuver the aircraft attitude and inertial velocity components, as well as UGV longitudinal velocity, can also be seen in Figure 5.

Finally, a stochastic analysis of the landing was performed, and Monte Carlo analysis [29] was used for this purpose. A total of 1000 simulations were made. The input variables mean values and variances were chosen based on previous data obtained during experiments as presented in [10]:

- Initial longitudinal displacement: mean of $166 \mathrm{~m}$, standard deviation of $10 \mathrm{~m}$

- Initial lateral displacement: mean of $0 \mathrm{~m}$, standard de-
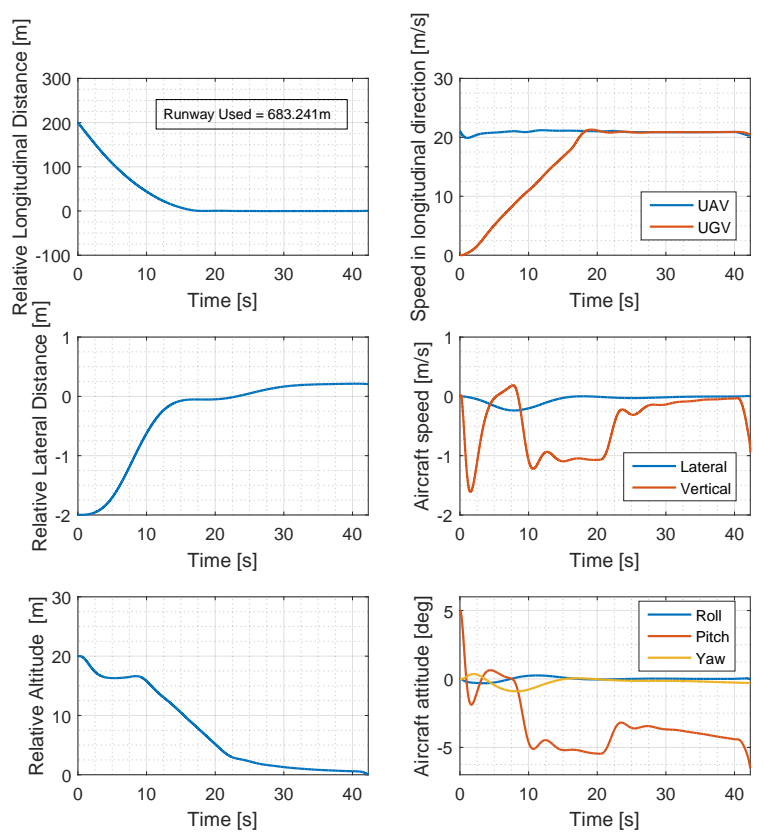

Fig. 5: Relative distances during the nominal landing procedure, as well as inertial velocities and aircraft attitude.

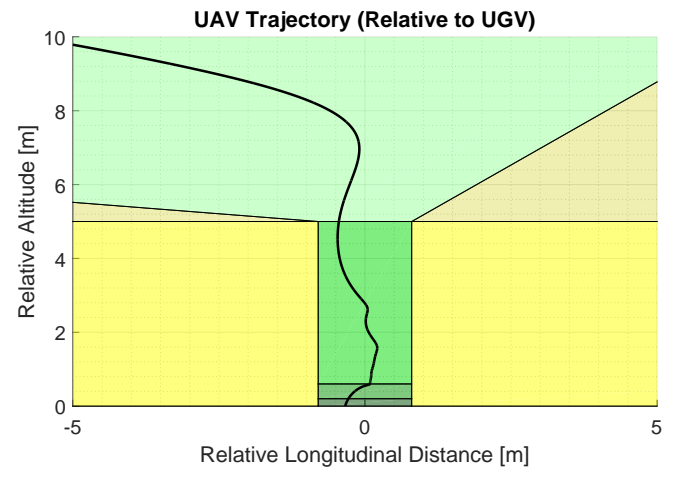

Fig. 6: Projected $x z$ trajectory in the UGV body-frame in a $10 \mathrm{~m} \times 10 \mathrm{~m}$ window centered in the middle of the landing platform during nominal landing.

viation of $6.6 \mathrm{~m}$

- Initial aircraft altitude: mean of $24 \mathrm{~m}$, standard deviation of $1 \mathrm{~m}$

- Initial aircraft groundspeed: mean of $21 \mathrm{~m} / \mathrm{s}$, standard deviation of $0.5 \mathrm{~m} / \mathrm{s}$

- Communication time delay (one-way): mean of $100 \mathrm{~ms}$, standard deviation of $50 \mathrm{~ms}$

- Scale factor for turbulence estimations: mean of 1 , standard deviation of 0.2

- Background wind intensity: mean of $3 \mathrm{~m} / \mathrm{s}$, standard deviation of $1 \mathrm{~m} / \mathrm{s}$

As outputs, the terminal position and velocity errors were considered, together with the runway used and time to land. Figures 7 and 8 illustrate the results. As can be seen in Figure 7a, all position errors lie within the $80 \mathrm{~cm}$ limit previously established, showing that in the defined conditions 
the aircraft can land safely. In Figure $7 b$, the mean value for the error in longitudinal velocity is positive due to the previously mentioned engine retard and slowdown of the aircraft, while the touchdown velocity exhibits values of around $-1 \mathrm{~m} / \mathrm{s}$, which was a design goal. Lastly, as seen in Figure 8 , an average of 43 seconds are necessary to complete the landing maneuver, and in terms of total runway used, $96 \%$ of data can be found between $633 \mathrm{~m}$ and $959 \mathrm{~m}$, and that number never exceeds $1 \mathrm{~km}$, which makes the landing comfortably feasible on the considered runway which is $2 \mathrm{~km}$ long.
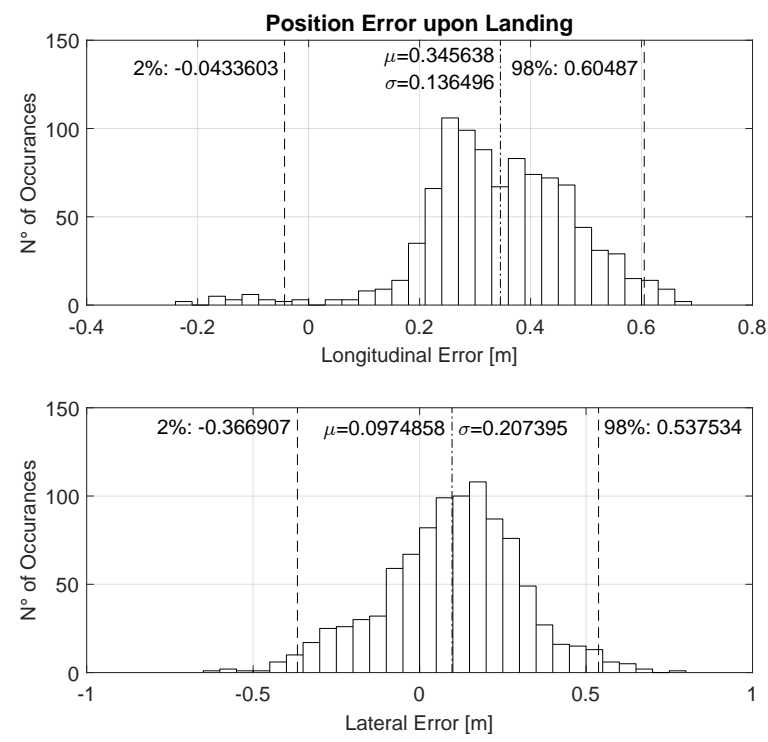

(a) Histogram of the horizontal position error upon touchdown.
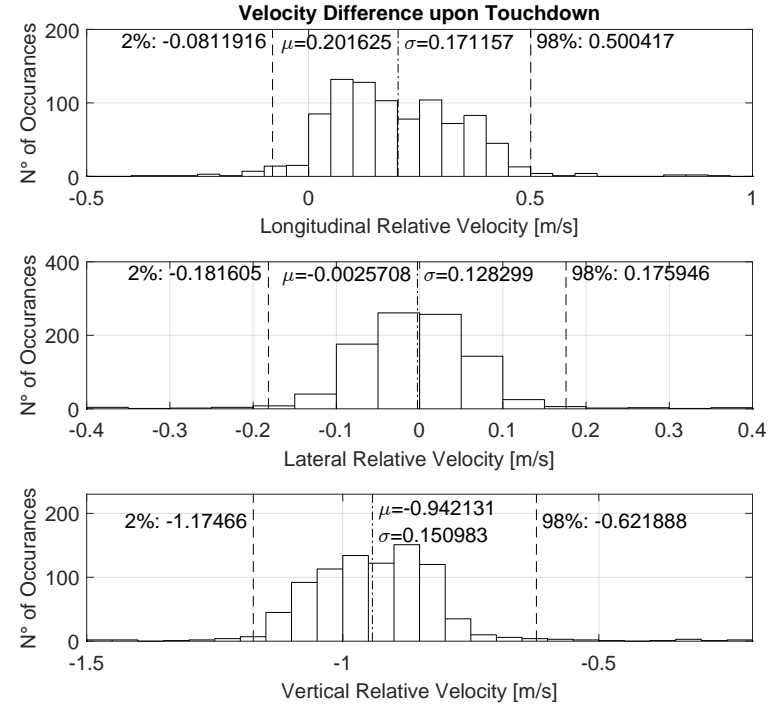

(b) Histogram of horizontal velocity error and relative vertical velocity on touchdown.

Fig. 7: Outputs of the position and velocity errors obtained through Monte Carlo analysis for the cooperative landing maneuver.
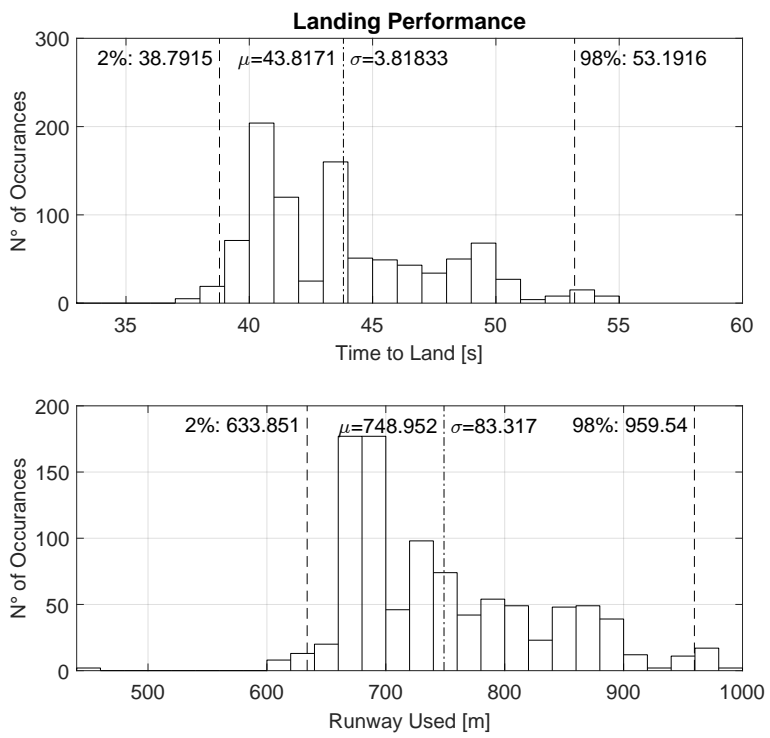

Fig. 8: Outputs of the landing performance on the Monte Carlo analysis for the cooperative landing maneuver.

\section{CONCLUSIONS}

In this work, the feasibility of a cooperative landing strategy for HALE UAVs was analyzed using both deterministic and stochastic methods for the examination of disturbance rejection capabilities and full landing system performance, producing good results, showing promise of the strategy for future applications. Therefore, optimal sets of controller values were estimated for UAV, UGV and the cooperative controller, which coordinates both vehicles during the procedure, using a methodology which was already validated on the smaller Penguin BE UAV to produce good results during real flight experiments [11].

As an important step, the system identification process was used to develop a flight dynamics model of the Elektra 2 Solar aircraft using a minimal set of aerodynamic model parameters, which proved to be high fidelity by FAA standards. Furthermore, it was shown that the developed model structure yields consistent results even with different sets of data.

One possible improvement to be made for future testing consists in the use of a faster ground vehicle; as previously mentioned, in this analysis, a slow vehicle model was used. With faster UGV dynamics, it would be easier to reject disturbances. Also, for an aircraft with such slow dynamics as Elektra 2 Solar, it makes sense to use an actuated landing platform with fast dynamics. The ElektRail [30] project (funded by the German aeronautics research program LuFo VI) aims to do exactly that, by creating a heavy duty rail system with fast dynamics to allow for the landing of a HALE aircraft with up to $600 \mathrm{~kg}$ takeoff mass on top of it. The framework created for the procedure shown in this article can easily be adapted and used in the scope of the ElektRail project, which might allow for tests with a real aircraft and platform in the near future. 
Another possible development step consists in the use of a different cooperative control strategy, as proposed in [13], where both vehicles act in both directions, while applying the technique presented in [31] used to make it robust to communication delays.

\section{REFERENCES}

[1] Romeo G., Frulla G., and Cestino E., "Design of a high-altitude longendurance solar-powered unmanned air vehicle for multi-payload and operations," Proceedings of the Institution of Mechanical Engineers, Part G: Journal of Aerospace Engineering, pp. 199 - 216, 2006.

[2] Maguire Y., "High altitude connectivity: The next chapter." Available at: https://engineering.fb.com/2018/06/27/ connectivity/high-altitude-connectivity-the - next-chapter/, 2017. Accessed on: 24.06.2020.

[3] Bleicher A., "Introducing Solara, the Atmospheric Satellite." Available at: https://spectrum.ieee.org/aerospace/aviation/ introducing-solara-the-atmospheric-satellite, 2013. Accessed on: 24.06.2020.

[4] NASA Dryden Flight Research Center, "Helios Prototype." Available at: https://www.nasa.gov/centers/dryden/news/ ResearchUpdate/Helios/. Accessed on: 24.06.2020.

[5] Airbus, "Zephyr Pioneering the Stratosphere." Available at: https: //www.airbus.com/defence/uav/zephyr.html. Accessed on: 24.06.2020.

[6] SFL GMBH, "ELHASPA." Available at: http://www . sfl-gmbh.de/en/blog/projekte/elhaspa/. Accessed on: 24.06.2020.

[7] "The National Transportation Safety Board (2016). Accident report, aircraft: Facebook UK ITD Aquila, registration: N565aq, ntsb identification: Dca16ca197." https://www.ntsb. gov/_layouts/ntsb.aviation/brief.aspx?ev_id= $20160701 \times 62525 \& k e y=1$.

[8] "The National Transportation Safety Board (2015). Accident report, aircraft: Titan aerospace holdings inc solara 50, registration: N950ta." https://www.ntsb.gov/_layouts/ntsb. aviation/brief.aspx?ev_id=20150505X85410\&key=1.

[9] Jones R. I., "The design challenge of high altitude long endurance (HALE) unmanned aircraft," The Aeronautical Journal (1968), 1999.

[10] Muskardin T., Balmer G., Wlach S., Kondak K., Laiacker M., and Ollero A., "Landing of a fixed-wing UAV on a mobile ground vehicle," 2016 IEEE International Conference on Robotics and Automation (ICRA), pp. 1237-1242, 2016.

[11] Muskardin, T., Balmer, G., Persson, L., Wlach, S., Laiacker, M., Ollero, A., and Kondak, K., "A novel landing system to increase payload capacity and operational availability of high altitude long endurance UAVs." Journal of Intelligent \& Robotic Systems, 88(24):597-618, 2017.

[12] Lütjens K. H., Lau A., Pfeiffer T., Loth S., Gollnick V., Klimek H., Greßmann B., Löwa S., and Blank C., "Airport2030-Lösungen für den effizienten Lufttransport der Zukunft," Deutsche Gesellschaft für Luftund Raumfahrt-Lilienthal-Oberth eV, 2012.

[13] Muskardin T., "Autonomous Landing of Fixed-Wing Aircraft on Mobile Platforms." PhD thesis, University of Seville, 2020.

[14] Beard R. W. and McLain T. W., "Small Unmanned Aircraft: Theory and Practice." USA: Princeton University Press, 2012.

[15] Stevens B. L., Lewis F. L., and Johnson E. N., "Aircraft Control and Simulation: Dynamics, Controls Design, and Autonomous Systems." Wiley-Blackwell, 3 ed., 2015.

[16] Brandt J. and Selig M., "Propeller Performance Data at Low Reynolds Numbers." American Institute of Aeronautics and Astronautics (AIAA), 2012.

[17] Klöckner A., "Geometry based flight dynamics modelling of unmanned airplanes," AIAA Modeling and Simulation Technologies Conference, 082013.

[18] Jategaonkar R. V., "Flight Vehicle System Identification: A TimeDomain Methodology." American Institute of Aeronautics and Astronautics (AIAA), 2 ed., 2015.

[19] Mulder J., Chu Q., Sridhar J., Breeman J., and Laban M., "Non-linear aircraft flight path reconstruction review and new advances," Progress in Aerospace Sciences, vol. 35, no. 7, pp. 673 - 726, 1999.

[20] de Ockham G., Ockham W., Boehner P., and Brown S., "Philosophical Writings: A Selection." Hackett Classics Series, Hackett Publishing Company, 1990.
[21] U.S Department of Transportation, "Airplane Simulator Qualification (AC 120-40C)," Federal Aviation Administration (FAA), 1995.

[22] Kong, J., Pfeiffer, M., Schildbach, G., and Borrelli, F. (2015). "Kinematic and dynamic vehicle models for autonomous driving control design.” In Intelligent Vehicles Symposium (IV), 2015 IEEE, pages 1094-1099. IEEE.

[23] Astrom K. J. and Murray R. M., "Feedback System: An Introduction for Scientists and Engineers." Princeton University Press, $2.11 \mathrm{~b}$ ed., 2012.

[24] Lambregts A., "Vertical flight path and speed control autopilot design using total energy principles." American Institute of Aeronautics and Astronautics (AIAA)

[25] de Sá Marques W. J., "Analysis and Comparison of Cooperative Control Architectures for Landing a Small Fixed-Wing Aircraft on a Mobile Platform," Bachelor's thesis, 2019.

[26] Skogestad S. and Postlethwaite I., "Multivariable Feedback Control: Analysis and Design." Wiley, 2005.

[27] Ogata K., "Modern Control Engineering." Instrumentation and controls series, Prentice Hall, 2010.

[28] Dorf R. and Bishop R., "Modern Control Systems." Prentice Hall, 2001.

[29] Fishman G., "Monte Carlo: Concepts, Algorithms, and Applications." Springer Series in Operations Research and Financial Engineering, Springer New York, 2013.

[30] RWTH Aachen Univerisity, Chair and Institute of Flight System Dynamics, "ElektRail", 2021. https://www.fsd. rwth-aachen.de/cms/fsd/Forschung/Projekte/ nnrrz/ElektRail/lidx/1/

[31] Muskardin, T., Coelho, A., Della Noce, E.R., Ollero, A., Kondak, K., "Energy-Based Cooperative Control for Landing Fixed-Wing UAVs on Mobile Platforms Under Communication Delays", IEEE Robotics and Automation Letters, 2020. 